\title{
THE MAGNETIC PROPERTIES AND THE BARKHAUSEN NOISE OF THE HYDROGENATED Fe-V-B AMORPHOUS ALLOY
}

\author{
L. CENIGA ${ }^{a *}$ AND L. NovÁK ${ }^{b \dagger}$ \\ ${ }^{a}$ Institute of Experimental Physics, Slovak Academy of Sciences \\ Watsonova 47, 04353 Košice, Slovak Republic \\ ${ }^{b}$ Department of Physics, Technical University \\ Komenského park 2, 04120 Košice, Slovak Republic
}

\begin{abstract}
As a consequence of the hydrogenation-dehydrogenation process, in the present paper the study of structural changes which could be followed by measuring structure sensitive magnetic properties as the stress induced anisotropy, the coercive force, the demagnetizing factor, and the Barkhausen noise parameters of the as-cast and hydrogenated $F_{80} V_{5} B_{15}$ amorphous alloys is performed.
\end{abstract}

PACS numbers: $61.43 . \mathrm{Dq}, 75.50 . \mathrm{Kj}, 75.60 . \mathrm{Ej}$

\section{Experimental method}

A sample of the $\mathrm{Fe}_{80} \mathrm{~V}_{5} \mathrm{~B}_{15}$ amorphous alloy was prepared by the spinning wheel technique in a form of the $10 \mathrm{~mm}$ wide and $20 \mu \mathrm{m}$ thick ribbon. The $150 \mathrm{~mm}$ long sample was hydrogenated electrolytically at room temperature for 2 hours $[1,2]$. The sample dehydrogenation was effected spontaneously in air at room temperature. The first series of measurements began with a measurement of magnetic parameters [1, 2] and Barkhausen noise parameters [3] in the as-cast state and then the sample was hydrogenated. The hydrogenation time is given in Figs. 1-3 and 6 as negative time on the time axis. The magnetic parameters were then measured at the interval of 30 minutes during 20 hours.

The measured magnetic parameters were: the whole quasi-static hysteresis curve and the anhysteresis curve from which the values of the saturation and remanent magnetic polarization, $J_{\mathrm{s}}$ and $J_{\mathrm{r}}$, respectively, the coercive force, $H_{\mathrm{c}}$, the total and stress induced anisotropy energy, $K_{i}$ and $K_{\sigma}[1,2]$, respectively, the total demagnetizing factor, $D$, as a sum of the inner and geometrical demagnetizing factor, $D_{\mathrm{i}}$ and $D_{\mathrm{g}}$ [2], respectively, were evaluated. $D_{\mathrm{g}}$ was calculated from the sample geometry [2], $D_{\mathrm{g}}=14.166 \times 10^{-5}$.

*e-mail: ceniga@saske.sk

†e-mail: novak@saske.sk 
The measured Barkhausen noise parameters were: the number of the Barkhausen pulses (BP) per a volume unit, $n$, which were registered during the magnetization process along one branch of the hysteresis loop, the total number of the BP per a volume unit, $N$, which represents the $n$ measured after the magnetization process, the power spectrum, $S(f)$ [3], and the relative amplitude distribution of BP, $N_{U} / N$, where $N_{U}$ represents the number of BP of the size $U$ per a volume unit, $N_{U}=\partial N / \partial U$. Using a single-layer pick-up coil of 100 turns and $10 \mathrm{~mm}$ length, BP amplifying was $15000 \times$ at which the output noise of the equipment used for the BP measurement was about $118 \mathrm{mV}$ (Fig. 7) [3].

\section{Results and discussion}

Hydrogen atoms which occupy Bernal's holes in the amorphous matrix [1] and increase inner stress are causing the rapid increase in $H_{\mathrm{c}}, K_{i}, K_{\sigma}, J_{\mathrm{s}}, J_{\mathrm{r}}$ (Figs. 1-3) [1, 2]. The chemical components of the $\mathrm{Fe}_{80} \mathrm{~V}_{5} \mathrm{~B}_{15}$ sample have not had a high affinity to the hydrogen atoms which then could move in the sample and could escape of the sample easily $[1,2]$. It could be then postulated that most of the hydrogen atoms left the sample at room temperature during 20 hours (Fig. 1-3) [1, 2]. The decreasing hydrogen content was then a reason of the magnetic parameters decrease $[1,2]$.

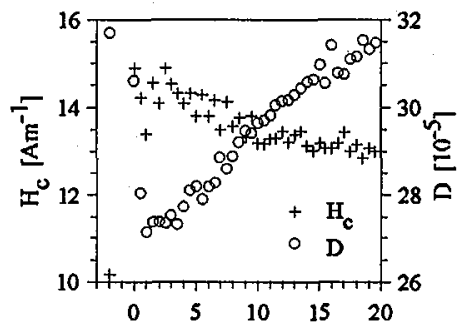

Fig. 1 Dehydrogenation time [h]

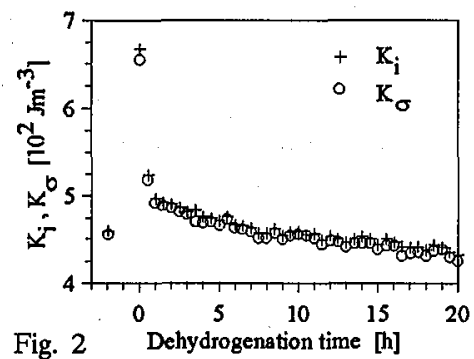

Fig. 2 Dehydrogenstion time [h]

Fig. 1. The coercive force, $H_{\mathrm{c}}$, and the total demagnetizing factor, $D$, vs. dehydrogenation time.

Fig. 2. The total and stress induced anisotropy energy, $K_{i}$ and $K_{\sigma}$, respectively, vs. dehydrogenation time.

After 20 hours of dehydrogenation $H_{\mathrm{c}}$ and $J_{\mathrm{s}}$ reached higher values than before hydrogenation. This fact could indicate that either the hydrogen atoms caused some defects remained in the structure during and after dehydrogenation, e.g. microcracks [3], or the rest of the hydrogen atoms remained in the sample even after 20 hours of dehydrogenation $[1,2]$. The $K_{i}-K_{\sigma}$ difference was almost constant and small, so it could be concluded that $K_{i}$ was mainly of the origin of $K_{\sigma}$ [1]. The small irreversible decrease in $K_{i}, K_{\sigma}, J_{\mathrm{r}}$ after 20 hours of dehydrogenation could correspond to the relaxation-like effect [1].

The course of $D$ during the hydrogen absorption and desorption processes is shown in Fig. 1. Modifying the Fe-V-B structure by hydrogenation, $D_{g}$ was unchanged, so the $D$ change was only due to the $D_{\mathrm{i}}$ change [2]. $D_{\mathrm{i}}$ is given by 


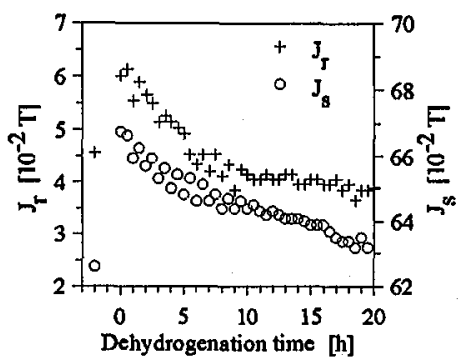

Fig. 3. The saturation and remanent magnetic polarization, $J_{\mathrm{s}}$ and $J_{\mathrm{r}}$, respectively, vs. dehydrogenation time.
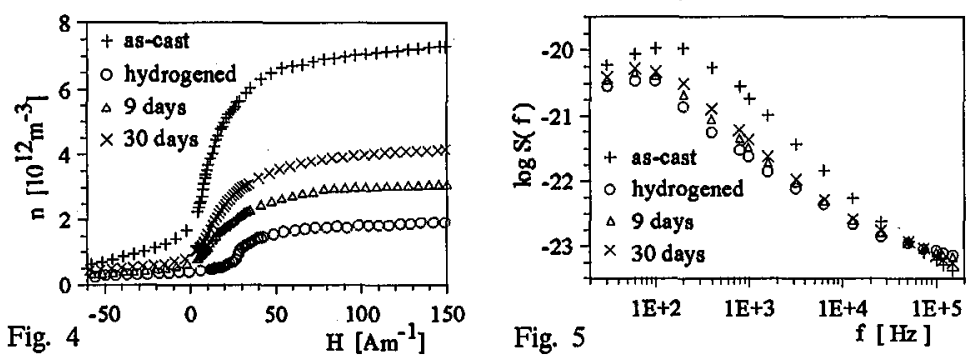

Fig. 4. The number of the Barkhausen pulses per a volume unit, $n$.

Fig. 5. The Barkhausen noise power spectrum, $S(f)$.

the demagnetizing field due to magnetic inhomogeneities inside the material, e.g. non-magnetic inclusions or cluster [2]. The $D$ decrease observed immediately after hydrogenation could then indicate that in the $\mathrm{Fe}-\mathrm{V}-\mathrm{B}$ structure there were weakly coupled $\gamma_{1}$-like antiferromagnetic clusters of the Fe atoms [2]. The $\gamma_{1}$-like antiferromagnetic clusters transformed to the $\gamma_{2}$-like ferromagnetic clusters $\left(J_{\mathrm{s}}\right.$ increases and $D$ decreases) $[2,4]$. This could be caused by changes of distances between the $\mathrm{Fe}$ atoms of the clusters as a consequence of the inner stress increase $[2,4]$. During dehydrogenation the $\gamma_{1}$-like antiferromagnetic clusters were probably reformed which could be indicated by the $D$ decrease [2]. The parameters shown in Figs. 1-3 were measured every day during 30 days of dehydrogenation but no rapid changes of their courses were observed.

Figure 4 shows the courses of $n$ during the magnetization process for the as-cast and hydrogenated states of the sample and after 9 and 30 days of dehydrogenation. The external magnetic field intensity, $H$, was the linear function of time, so $n$ was also time dependent which means that $\mathrm{d} n / \mathrm{d} t \approx \mathrm{d} n / \mathrm{d} H . S(f)$ (Fig. 5), $S(f) \approx \mathrm{d} n / \mathrm{d} t$ [32], has been always registered at the external field intensity being equal to the sample coercive force. The differences of the $n$ gradient at $H=H_{c}$ for the measured sample states were then responsible for the expressive differences of the $S(f)$ intensity [3].

The course of $N / N_{\text {ac }}$ during dehydrogenation is shown in Fig. 6 for the as-cast sample $N_{\mathrm{ac}}=0.74 \times 10^{13} \mathrm{~m}^{-3}$ and the course of the relative amplitude distribution 

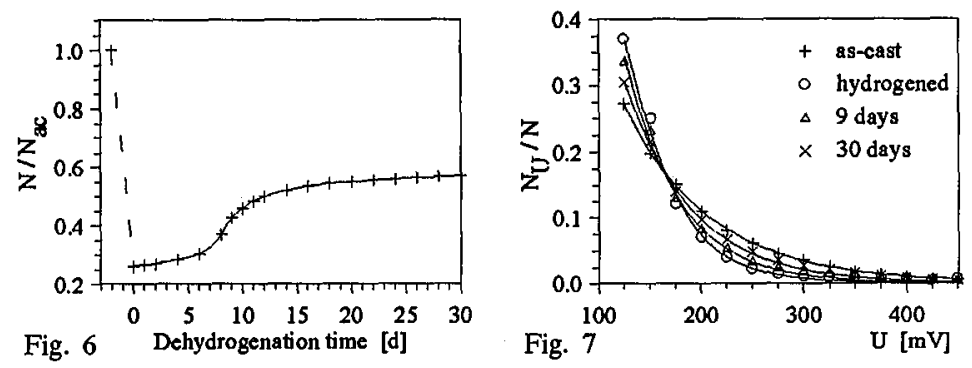

Fig. 6. The $N / N_{\text {ac }}$ parameter vs. dehydrogenation time.

Fig. 7. The relative amplitude distribution of the Barkhausen pulses, $N_{U} / N$.

of $\mathrm{BP}, N_{U} / N$, is shown in Fig. 7. The Barkhausen noise of the hydrogenated sample was given by a higher rate and a smaller rate of low and middle BP (Fig. 7), respectively. The higher rate and the smaller rate could correspond to the more convex course of $S(f)$ in the $0.2-50 \mathrm{kHz}$ frequency range and the $S(f)$ increase above $50 \mathrm{kHz}$ for the hydrogenated sample (Fig. 5), respectively [3]. BP of about $175 \mathrm{mV}$ kept their rate in the Barkhausen noise constant which could be probably connected with the $S(f)$ course at $50 \mathrm{kHz}[3]$.

$n, S(f), N / N_{\mathrm{ac}}$, and $N_{U} / N$ changed rapidly after hydrogenation as a consequence of the inner stress increase but the time delay of this parameters reaction to dehydrogenation is observed. After the inner stress reduction during 20 hours of dehydrogenation, the rest of the hydrogen atoms could be probably more stabilized in the amorphous matrix and in the microcracks which perhaps rose by hydrogenation [3]. This stabilization could explain almost constant course of $n$, $S(f), N / N_{\text {ac }}, N_{U}$, and $N_{U} / N$ up to 6-7 days after hydrogenation. The expressive changes of these parameters after 6-7 days could be perhaps caused by a more intensive dehydrogenation although no expressive changes of the magnetic parameters shown in Figs. 1-3 were observed. This contradiction has not been probably published so far, and then it would require a much more detailed research. The presence of the hydrogen atoms rest $[1,2]$ and perhaps the microcracks [3] could be responsible for the irreversible changes of $n, S(f), N / N_{\mathrm{ac}}, N_{U}$, and $N_{U} / N$ observed even after 30 days of dehydrogenation.

\section{Acknowledgments}

This work was supported by the Slovak Grant Agency VEGA (No. 2/5140/98).

\section{References}

[1] L. Novák, É. Kisdi-Koszó, A. Stančáková, L. Kafková, M. Konč, Key Eng. Mater. 81-83, 337 (1993).

[2] L. Novák, É. Kisdi-Koszó, M. Konč, M. Zatroch, J. Magn. Magn. Mater. 160, 261 (1996).

[3] L. Ceniga, K. Šterbáková, L. Novák, M. Zentková, Czech. J. Phys. 49, 549 (1999).

[4] T. Kaneko, K. Shirakawa, S. Abe, T. Masumoto, J. Magn. Magn. Mater. 54-57, 305 (1986). 